\title{
Handover Queuing Strategies with Dynamic and Fixed Channel Allocation Techniques in Low Earth Orbit Mobile Satellite Systems
}

\author{
Enrico Del Re, Senior Member, IEEE, Romano Fantacci, Senior Member, IEEE, \\ and Giovanni Giambene, Member, IEEE
}

\begin{abstract}
This paper deals with the performance evaluation of various resource management strategies that are suitable for low earth orbit-mobile satellite systems (LEO-MSS's). A user mobility model has been proposed and its statistical parameters have been derived. Both fixed channel allocation (FCA) and dynamic channel allocation (DCA) techniques have been considered. Moreover, in order to reduce the handover failure probability, we have assumed that interbeam handover requests which do not immediately obtain service can be queued. In particular, two different queuing disciplines have been compared: a) the first input first output (FIFO) scheme and b) a new technique called last useful instant (LUI) which is based on the knowledge of the maximum time within which the handover procedure must be accomplished. Implementation aspects for the LUI technique in a LEO-MSS have been discussed also in comparison with the measurement-based priority scheme (MBPS), previously proposed in the literature on this subject. The efficiency of the LUI queuing scheme as regards the FIFO technique has been investigated by simulations for both DCA and FCA techniques. An analytical approach has been also presented in order to allow the performance evaluation of the FCA scheme with different handover queuing disciplines.
\end{abstract}

Index Terms - Dynamic channel allocation, satellite communications.

\section{INTRODUCTION}

$\mathbf{T}$ HE POTENTIALITY of the future cellular market is incredibly large, since half of the world's population lives more than two hours away from a telephone [1]. At present, $R \& D$ efforts are addressed toward the achievement of mobile services on a global basis. The integration of mobile satellite systems (MSS's) with terrestrial cellular networks [2]-[4] will pave the way for future third-generation mobile communication systems, named International Mobile Telecommunications after the year 2000 (IMT-2000) [5].

Several satellite orbital constellations have been proposed for MSS's. An interesting solution is given by low earth orbit (LEO) satellites, since they permit relaxation of the constraints on the link budget, allow the use of low-power handheld mobile terminals, and ensure the earth coverage with smaller cells, so achieving a higher traffic capacity [6]-[9].

Paper approved by D. Everitt, the Editor for Wireless Network Performance of the IEEE Communications Society. Manuscript received October 7, 1996; revised May 22, 1998 and July 20, 1998. This work was supported by the Italian Space Agency and MURST.

The authors are with the Dipartimento di Ingegneria Elettronica, Universitá degli Studi di Firenze, 50139 Firenze, Italy (e-mail: fantacci@lenst.die.unifi.it).

Publisher Item Identifier S 0090-6778(99)00794-1.
LEO satellites are not stationary with respect to a fixed point on the earth: the satellite ground-track speed $V_{t r k}$ is far greater than the earth rotation speed and the user speed [7]. Two different approaches are possible for the cellular coverage with LEO-MSS's: 1) cells (=spot-beam footprints on the earth) are fixed on the earth and satellite antenna spot-beams are steered so as to point to the same area during all the time the satellite is above the horizon (e.g., TELEDESIC system [9]) and 2) cells move on the earth according to the satellite motion (e.g., IRIDIUM system [7], [8]). This paper deals with the second solution that requires specific procedures to manage the cell change for an active call. Moreover, we consider only a voice traffic. Each time a call arrives at a cell $x$, it must be served by an available channel in $x$; call attempts that do not immediately find free resources are blocked and lost [blocked calls cleared (BCC)].

The main problem is that the spectrum assigned to LEOMSS's [10] is reduced with respect to the expected market diffusion of these services. Therefore, optimized radio resource management strategies have to be investigated for LEOMSS's. First, we have considered a fixed channel allocation (FCA) technique and we have developed an analytical approach to derive the system performance in this case. Moreover, we have considered dynamic channel allocation (DCA) techniques [11]-[15], because they are more efficient resource management strategies that can provide MSS's with a high capacity. Owing to analytical difficulties, the DCA performance has been derived only by simulations.

When an active mobile subscriber (MS) goes out from a cell and enters an adjacent one, a new channel must be automatically assigned to it in order to have a seamless conversation. This procedure is called handover; it involves the rerouting of a call between two adjacent beams that may belong to either the same satellite or two adjacent satellites of the MSS's. If no channel is available in the destination cell, the handover is unsuccessful and the call is dropped.

The selection of a suitable policy for managing handover requests is a central issue in defining resource management strategies. From the user standpoint, the interruption of a conversation is more undesirable than the blocking of a newly arriving call. In LEO-MSS's, interbeam handover requests are extremely frequent during call lifetime (one could expect that a call experiences a handover request every $1 \mathrm{~min}$ or even less) and at each beam change the call may be dropped due to an unsuccessful handover. Hence, LEO-MSS's require specific techniques that prioritize the service of handover requests 


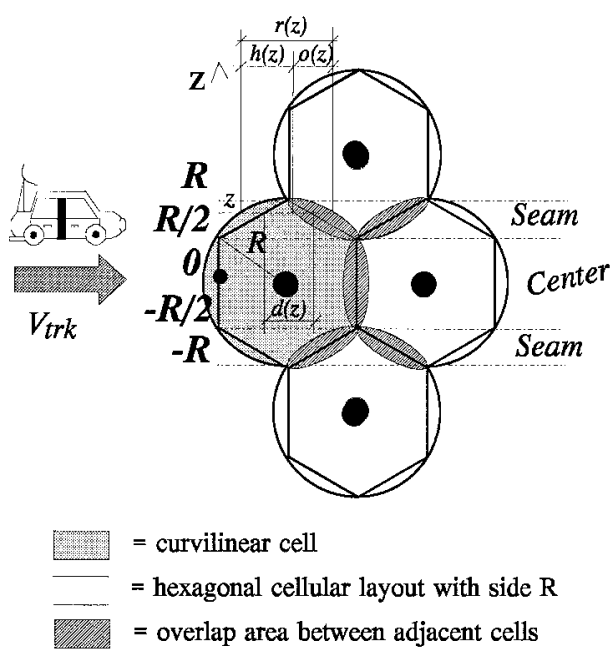

Fig. 1. The geometry of the cellular network with a hexagonal layout.

with respect to the service of new call attempts in order to reduce as much as possible the call dropping probability. Many policies have been recently proposed to privilege handovers at the expenses of new call arrivals in terrestrial microcellular systems [12], [16]-[21]. The aim of this paper is to present a suitable mobility model and to compare the performance of different handover queuing strategies in LEO-MSS's.

This paper has been organized as follows: Section II deals with some preliminary assumptions. Section III presents a suitable mobility model that has been analyzed in Section IV. Both FCA and DCA techniques are described in Section V. Section VI presents different handover queuing disciplines. An analytical study for the FCA technique with handover queuing is presented in Section VII. Finally, Section VIII deals with simulation results for both DCA and FCA.

\section{BASIC ASSUMPTIONS}

This paper utilizes IRIDIUM system data [8] as an example for numerical evaluations, but the results obtained here are generally valid for all LEO-MSS's based on the moving cells approach described in Section I. In particular, the IRIDIUM system is based on 66 satellites over six near polar circular orbits at about $780 \mathrm{~km}$ of altitude (consequently, $V_{t r k}=$ $26600 \mathrm{~km} / \mathrm{h}$ ).

Let us assume that spot-beam footprints on the earth have a regular shape due to beam-forming. Moreover, we consider that each multispot-beam antenna from a satellite irradiates on the earth a regular honeycomb cellular network, where $R$ is the cell side (Fig. 1). Therefore, the centers of adjacent cells are separated by a distance equal to $\sqrt{3} R$. In the IRIDIUM case, we have assumed $R$ equal to $212.5 \mathrm{~km}$ [14].

In this paper, we do not refer to a particular multiple access technique (TDMA, CDMA, FDMA); a channel is a resource shared among users according to allocation rules.

New calls are assumed to arrive at the cells according to a Poisson process independent of cell to cell, and the unencumbered call duration is considered exponentially distributed with average value $T_{m}$.

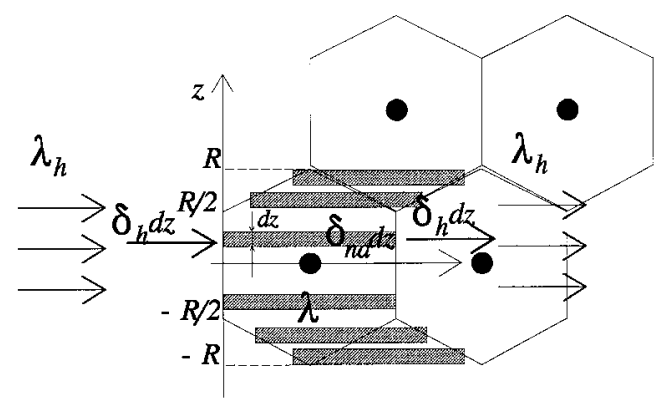

Fig. 2. The flow conservation for handover requests that enter and handover requests that go out from a cell.

According to the BCC policy for the voice traffic, the following quality of service (QoS) parameters are used to evaluate the performance of resource management strategies:

- the blocking probability of new call attempts $P_{b 1}$;

- the handover failure probability $P_{b 2}$;

- the call dropping probability $P_{\text {drop }}$;

- the unsuccessful call probability $P_{n s}$, because the call is initially blocked or it is dropped due to the failure of a subsequent handover request.

The QoS parameters directly experienced by MS's are $P_{b 1}$ and $P_{\text {drop. }}$. ITU-T in the recommendation E.771 [22] has specified that $P_{\text {drop }}$ and $P_{b 1}$ should not exceed $5 \cdot 10^{-4}$ and $10^{-2}$, respectively. These requirements, given for land mobile cellular systems, have been adopted here for LEO-MSS's, even if they may seem too severe, by considering future high-quality mobile communication systems.

Finally, only mobile-to-fixed user calls and fixed-to-mobile user calls have been taken into account, because mobile-tomobile user calls are expected to be a little percentage of the whole traffic (maximum 5\%).

\section{THE LEO MoBILITY MODEL}

In order to study the handover generation process toward a cell and evaluate the impact of handover strategies on the performance of resource management techniques, it is necessary to model the user mobility. Then, the following aspects have to be considered: 1) the propagation conditions in the satellite radio channel; 2) the user motion with respect to cells; and 3) the geometry of the cells and their disposition, that is the topology of the network. In order to reduce the analytical complexity of the model, a widely used approach [14], [17], [23] is to neglect propagation aspects; therefore, this study takes only into consideration the user motion and the network topology.

We name source cell the cell where the MS call starts and transit cell any subsequent cell reached by the MS with the call in progress. Referring to a given cell $x$, subscript $i=1$ will be used for the statistical parameters related to calls started in cell $x$, whereas subscript $i=2$ will be used for the parameters related to handed-over calls to cell $x$. We consider hexagonal regular cells with side $R$ (see Figs. 1 and 2); this assumption will allow us to obtain a simple analytical characterization of user mobility. 
The relative satellite-MS motion can be approximated by only the satellite ground-track speed (i.e., vector $V_{t r k}$ ), due to the high value of $V_{t r k}$ with respect to the other motion component speeds. This consideration also entails that the relative motion has a fixed orientation with respect to the cellular layout irradiated on the earth by satellites. Let the track of the relative motion be disposed, as shown in Fig. 1.

Moreover, MS's (and the calls they generate) are considered uniformly distributed ${ }^{1}$ over the simulation area [13]-[15]. Therefore, a newly arriving call can occur with equal probability in every point of the satellite cellular network.

According to the above assumptions, we have the following.

- When a new call arrival occurs, any cell of the system has the same probability to be the source cell of this arrival.

- Once the source cell of a call is defined, a random offset $z \in[-R, R]$ is associated to this call. ${ }^{2}$ The probability density function (pdf) of $z, f(z)$, is obtained by taking into account that active MS's are uniformly generated within a cell and they move in straight lines with fixed $z$ values. A new call in its source cell belongs to a horizontal elementary strip with side $d(z)$ and height $d z$ (see Fig. 2) according to a probability given by the ratio between the strip area $[=d(z) d z]$ and the cell area $\left(=3 \sqrt{3} R^{2} / 2\right)$. Then, $f(z)$ is given by

$$
f(z)=\frac{d(z)}{3 \frac{\sqrt{3}}{2} R^{2}}
$$

where $d(z)$ is

$$
d(z)= \begin{cases}\sqrt{3} R, & \text { if }|z| \leq \frac{R}{2} \\ 2 \sqrt{3}(R-|z|), & \text { if } R \geq|z|>\frac{R}{2} .\end{cases}
$$

- Once the offset $z$ of the MS is chosen in the source cell, the distance covered in this cell by the MS from the call arrival instant is uniformly distributed between zero and $d(z)$.

According to the uniform spatial generation for new call attempts, the offset $z$ of an MS in a transit cell is uniformly distributed. It follows that the pdf of the offset $z$ according to which an MS crosses the source cell $(i=1)$ or a transit cell $(i=2), f_{i}(z)$, can be obtained as

$$
f_{i}(z)= \begin{cases}f(z), & \text { if } i=1 \\ \frac{\{u[z+R]-u[z-R]\}}{2 R}, & \text { if } i=2\end{cases}
$$

where:

$$
u(x) \triangleq \begin{cases}1, & x \geq 0 \\ 0, & \text { otherwise }\end{cases}
$$

\footnotetext{
${ }^{1}$ Even if LEO-MSS's will be characterized by time-varying traffic conditions, we use the hypothesis of uniform traffic, because it allows generic performance evaluations which do not depend on specific traffic conditions (see also the next section). The interested reader may find more details on this subject in [3].

${ }^{2}$ If we refer to a satellite polar constellation (e.g., IRIDIUM) on the basis of previous assumptions on the disposition of the cellular layout with respect to the ground-track speed, we obtain that the coordinate $z$ in Figs. 1 and 2, here called offset, is related to the MS longitude.
}

We can summarize the proposed LEO mobility model as follows.

1) MS's cross the cellular network with a relative velocity (i.e., vector $V_{t r k}$ ), disposed as shown in Fig. 1 with respect to the cellular layout.

2) When a handover occurs, the destination cell will be the neighboring cell in the direction of the relative satelliteMS motion.

3) MS's cross the cellular network with an offset uniformly distributed all over the network.

4) From the call arrival in a cell, where $z$ is the offset of the related MS according to the reference shown in Fig. 1, the related MS travels a distance in this cell which is

- uniformly distributed between zero and $d(z)$ if the cell is the source cell of the call;

- deterministically equal to $d(z)$ if the cell is a transit cell of the call.

In order to characterize the user (relative) mobility in LEOMSS's, we introduce the dimensionless parameter $\alpha$ as

$$
\alpha \triangleq \frac{\sqrt{3} R}{V_{t r k} T_{m}}
$$

where $T_{m}$ is the average call duration.

Parameters $R$ and $V_{t r k}$ depend on the satellite constellation altitude; moreover, $R$ also depends on the half power beam width (HPBW) of the satellite antenna spot-beams. Typical $\alpha$ values are $0.20-0.60$ for LEO-MSS's, if $T_{m}=3 \mathrm{~min}$. The smaller $\alpha$ is, the more frequent the handover requests are during call lifetime: i.e., the mobility increases [see (25) and (26) in the next section]. In particular, in the IRIDIUM mobility case under examination $\left(R=212.5 \mathrm{~km}, V_{t r k}=\right.$ $26600 \mathrm{~km} / \mathrm{h}) \alpha$ is about equal to 0.27 , if $T_{m}=3 \mathrm{~min}$.

With respect to the previously proposed LEO mobility model [13]-[15], this model takes into account that an MS with a call in progress may cross the cellular layout not only along the central region of cells (see Fig. 1), but also through the seam of the cellular network. In such a case, we expect that the number of interbeam handovers during call lifetime is significantly increased. This entails a more realistic evaluation of the impact of user mobility on the performance of channel allocation techniques for LEO-MSS's. Finally, it is worth noting that the mobility model proposed in this paper is valid for whatever type of MS's, since $V_{t r k}$ is far greater than the speed of every kind of MS, it does not matter if it is pedestrian, vehicular, or flying in a plane.

\section{ANALysis OF THE LEO MoBILITy Model}

Let us refer to a given cell $x$. We denote by $t_{m c 2}$ the time spent by an MS to cross cell $x$ from border to border (i.e., the mobile sojourn time in a cell), and by $t_{m c 1}$ the time spent in cell $x$ by an MS from the new call arrival instant. The distributions of variables $t_{m c i}$ can be easily derived as

$$
\operatorname{Prob}\left\{t_{m c i}<t\right\}=\int_{-R}^{R} \operatorname{Prob}\left\{t_{m c i}<t \mid z\right\} f_{i}(z) d z
$$


where

$$
\begin{aligned}
& \operatorname{Prob}\left\{t_{m c i}<t \mid z\right\} \\
& =\left\{\begin{array}{cc}
\frac{t V_{t r k}}{d(z)}\left\{u(t)-u\left[t-\frac{d(z)}{V_{t r k}}\right]\right\} & \text { if } i=1 ; \\
+u\left[t-\frac{d(z)}{V_{t r k}}\right], & \text { if } i=2 . \\
u\left[t-\frac{d(z)}{V_{t r k}}\right], &
\end{array}\right.
\end{aligned}
$$

The pdf of variables $t_{m c i}$ are obtained by taking the derivative of (6) with respect to variable $t$. Through some algebraic manipulations we have

$$
\operatorname{pdf}_{t_{m c 1}}(t)=\frac{2}{3}\left\{2-\frac{t}{\alpha T_{m}}\right\} \frac{\left\{u(t)-u\left(t-\alpha T_{m}\right)\right\}}{\alpha T_{m}}
$$

and

$$
\operatorname{pdf}_{t_{m c 2}}(t)=\frac{\left\{u(t)-u\left(t-\alpha T_{m}\right)\right\}}{2 \alpha T_{m}}+\frac{1}{2} \delta\left(t-\alpha T_{m}\right)
$$

where $\delta(t)$ is the Dirac delta function.

The expressions for the distributions of the random variables $t_{m c 1}$ and $t_{m c 2}$ are not independent of each other, because $t_{m c 1}$ can be considered as a residual time in the interval $t_{m c 2}$ starting from an arrival instant within $t_{m c 2}$ (excess life theorem [19], [24], [25]). Hence, $\operatorname{pdf}_{t_{m c 1}}(t)$ and $\operatorname{pdf}_{t_{m c 2}}(t)$, respectively, given by (8) and (9), can be related as

$$
\operatorname{pdf}_{t_{m c 1}}(t)=\frac{1-\int_{\tau=0}^{t} \operatorname{pdf}_{t_{m c 2}}(\tau) d \tau}{E\left[t_{m c 2}\right]} .
$$

Since the unencumbered call duration $t_{d}$ is exponentially distributed, the same distribution is valid for the residual call lifetime after a handover request (memoryless property). Therefore, we still denote by $t_{d}$ the residual call lifetime.

A handover procedure is started for an MS with a call in progress in cell $x$ whenever $t_{d}>t_{m c i}$, with $i=1$ if cell $x$ is the source cell for the call, or $i=2$ if cell $x$ is a transit cell for the call. The probabilities of these events, $P_{H i}, i=1,2$, can be obtained as follows:

$$
\begin{aligned}
P_{H i} & =\operatorname{Prob}\left\{t_{d}>t_{m c i}\right\} \\
& =\int_{0}^{+\infty} \operatorname{Prob}\left\{t_{d}>t \mid t_{m c i}=t\right\} \operatorname{pdf}_{t_{m c i}}(t) d t \\
& =\int_{0}^{+\infty} e^{-\left(t / T_{m}\right)} \operatorname{pdf}_{t_{m c i}}(t) d t \\
& =\left.\mathcal{L}\left(\operatorname{pdf}_{t_{m c i}}(t)\right)\right|_{s=1 / T_{m}} \quad i=1,2
\end{aligned}
$$

where $\mathcal{L}(g(t))$ denotes the Laplace transform of the function $g(t)$ [24].

By substituting (8) and (9) into (11) and by performing some algebraic manipulations, we obtain the handover probabilities $P_{H 1}$ and $P_{H 2}$

$$
\begin{aligned}
P_{H 1}(\alpha) & =\frac{2}{3}\left\{P_{h 1}(\alpha)+\frac{1-P_{h 1}(\alpha)}{\alpha}\right\} \\
P_{H 2}(\alpha) & =\frac{P_{h 1}(\alpha)+P_{h 2}(\alpha)}{2}
\end{aligned}
$$

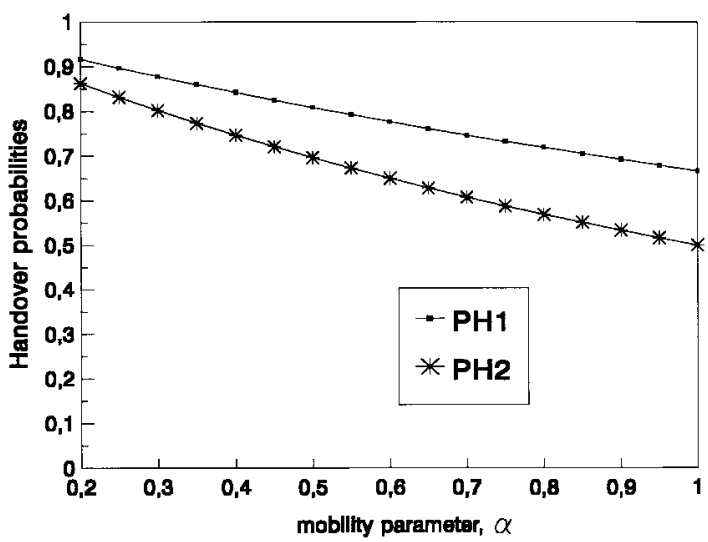

Fig. 3. Handover probabilities $P_{H 1}$ and $P_{H 2}$ as a function of the mobility parameter $\alpha$

where

$$
P_{h 1}(y) \triangleq \frac{1-e^{-y}}{y}, \quad P_{h 2}(y) \triangleq e^{-v} .
$$

Note that $P_{h i}(y)$ in (13), $i=1,2$, represents the handover probability for a call (with mean duration $T_{m}$ ) from a cell, where the related MS crosses a distance (from the call arrival instant) which is:

- uniformly distributed between zero and $q$ for $i=1$;

- fixed and equal to $q$ for $i=2$

where $y=q /\left[V_{t r k} T_{m}\right]$. More details are given in [14].

Handover probabilities $P_{H 1}$ and $P_{H 2}$ only depend on the mobility parameter $\alpha$. Fig. 3 shows the behaviors of $P_{H 1}$ and $P_{H 2}$ as a function of $\alpha$. It is evident that as $\alpha$ approaches zero $(\infty), P_{H 1}$ and $P_{H 2}$ approach one (zero), i.e., the mobility increases (decreases).

The channel holding time for calls in cell $x$ can be derived as [17]

$$
t_{H i}=\min \left[t_{d}, t_{m c i}\right], \quad i=1,2 .
$$

The statistical distribution of variable $t_{H i}, i=1,2$, can be derived by means of the approach outlined in [17]. For the sake of brevity, we give below only the final result for the expected value of $t_{H i}$ [24]

$$
E\left[t_{H i}\right]=T_{m}\left(1-P_{H i}\right), \quad i=1,2 .
$$

Equation (15) shows that, due to mobility, the average channel holding time in a cell (both source cell and transit cell) is reduced with respect to $T_{m}$.

We would like to point out that in this paper the performance evaluation of different handover strategies has been limited to the uniform traffic case, because it allows us to focus on the more generic aspects of the system rather than obtaining results for very specific traffic profiles. However, our study can be extended to the case of nonuniform traffic on the condition that a suitable traffic distribution be provided. The same authors have presented in [25] a traffic model based on a market forecast for future IMT-2000 [see Fig. 11(a)]. With reference to that traffic model, we have shown in Fig. 11(b), Section VIII, a performance comparison in terms of $P_{n s}$ between FCA and DCA both employing the queuing of handover 
requests (see Sections V and VI). As expected, the advantages of the DCA technique are enhanced in this case. A further investigation on this point is beyond the scope of this paper.

According to the uniform traffic assumption, we denote by $\lambda$ the average arrival rate of new call attempts in a generic cell of the system. The handover arrival process examined here is related to calls managed by the MSS which need to be passed from cell to cell: i.e., interbeam handovers. The handover arrival process in a cell depends on both the motion of MS's as regards the cells, the shape and size of cells, and MS's distribution on the territory. Since all cells have the same shape and size by means of beamforming, all MS's have the same motion conditions as regards the cells (due to the high value of the satellite ground-track speed). Moreover, the MS distribution on the territory is uniform and the average handover arrival rate toward a cell, $\lambda_{h}$, is the same for all the cells (see Fig. 2). We obtain a relationship between $\lambda_{h}$ and $\lambda$ by application of the flow balance condition among outgoing handover requests and incoming handover requests in a given area [14]. In particular, we consider flow conservation equations on horizontal strips with elementary height $d z$ and length $\sqrt{3} R$ that are disposed on the cellular network at offsets $z$, from $z=-R$ to $z=R$ (see Fig. 2). The elementary arrival rates in a generic strip are $\delta_{n a}(z) d z$ and $\delta_{h}(z) d z$ for new call attempts and handover requests, respectively. The sum of the elementary rates $\delta_{h}(z) d z$ for the strips from $z=-R$ to $z=R$ gives the average arrival rate of handover requests toward a cell

$$
\int_{-R}^{R} \delta_{h}(z) d z=\lambda_{h}
$$

The sum of the areas of the elementary strips from $z=-R$ to $z=R$ is equal to $4 / 3$ the area of a cell. Due to the uniform spatial generation of new call arrivals, $\delta_{n a}$ is independent of $z$. Hence, we have

$$
\int_{-R}^{R} \delta_{n a} d z=\frac{4}{3} \lambda \quad \Longrightarrow \quad \delta_{n a}=\frac{2}{3 R} \lambda
$$

In the following analysis, we will distinguish the case $|z| \leq$ $R / 2$ from the case $R \geq|z|>R / 2$.

1) Case $|z| \leq R / 2$ : The flow balance condition is analogous to that shown in [14], but instead of parameters $\lambda$ and $\lambda_{h}$, we have to consider terms $\delta_{n a} d z$ and $\delta_{h}(z) d z$ which are related to a generic elementary strip at the offset $z$

$\delta_{n a} d z\left(1-P_{b 1}\right) P_{h 1}(\alpha)+\delta_{h}(z) d z\left(1-P_{b 2}\right) P_{h 2}(\alpha)=\delta_{h}(z) d z$.

Let $\lambda_{h c}$ denote the average handover rate for the central part of a cell defined as

$$
\lambda_{h c}=\int_{-R / 2}^{R / 2} \delta_{h}(z) d z
$$

Through integration of (18) from $z=-R / 2$ to $z=R / 2$ and by using (17) and (19), we have

$$
\frac{\lambda_{h c}}{\lambda}=\frac{2}{3} \frac{\left(1-P_{b 1}\right) P_{h 1}(\alpha)}{1-\left(1-P_{b 2}\right) P_{h 2}(\alpha)} .
$$

2) Case $R \geq|z|>R / 2$ : The cell border divides an elementary strip at the offset $z$ into two segments with length $d(z)$ and $\sqrt{3} R-d(z)$, respectively. Let us denote

$$
\alpha_{1}(z)=\frac{d(z)}{V_{t r k} T_{m}}, \quad \alpha_{2}(z)=\frac{\sqrt{3}-d(z)}{V_{t r k} T_{m}} .
$$

Therefore, we have $\alpha_{1}(z)+\alpha_{2}(z)=\alpha$.

Owing to the uniform spatial generation of new calls, a new call arrival (in the strip) occurs in the first segment with a probability $d(z) /(\sqrt{3} R)=\alpha_{1} / \alpha$ [see (21)] and in the second segment with a probability $[\sqrt{3} R-d(z)] /(\sqrt{3} R)=\alpha_{2} / \alpha$ [see (21)]. Consequently, a new call arrival originated in this strip gives rise to a handover request with a probability (1$\left.P_{b 1}\right)\left[\left(\alpha_{1} / \alpha\right) P_{h 1}\left(\alpha_{1}\right) P_{h 2}\left(\alpha_{2}\right)\left(1-P_{b 2}\right)+\left(\alpha_{2} / \alpha\right) P_{h 1}\left(\alpha_{2}\right)\right]$. Whereas a call handed-over toward the strip requires a new handover with a probability equal to $\left(1-P_{b 2}\right) P_{h 2}\left(\alpha_{1}\right)(1-$ $\left.P_{b 2}\right) P_{h 2}\left(\alpha_{2}\right)=\left(1-P_{b 2}\right)^{2} P_{h 2}(\alpha)$. Hence, we have the following flow balance equation:

$$
\begin{aligned}
\delta_{n a} d z\left(1-P_{b 1}\right) & {\left[\frac{\alpha_{1}}{\alpha} P_{h 1}\left(\alpha_{1}\right) P_{h 2}\left(\alpha_{2}\right)\left(1-P_{b 2}\right)+\frac{\alpha_{2}}{\alpha} P_{h 1}\left(\alpha_{2}\right)\right] } \\
& +\delta_{h}(z) d z\left(1-P_{b 2}\right)^{2} P_{h 2}(\alpha)=\delta_{h}(z) d z .
\end{aligned}
$$

Let us integrate both sides of (22) on the seam of the cellular network, i.e., from $z=-R$ to $z=-R / 2$ and from $z=R / 2$ to $z=R$. For the symmetry of the problem, this is equivalent to integrating both sides of (22) multiplied by two, from $z=R / 2$ to $z=R$. Moreover, we divide the result by $\lambda$ and we obtain $\lambda_{h} / \lambda$ as

$$
\begin{aligned}
\frac{\lambda_{h}}{\lambda}= & \frac{\lambda_{h c}}{\lambda}+\frac{2}{3}\left(1-P_{b 1}\right) \\
& \cdot \frac{1-P_{h 1}(\alpha)+\left(1-P_{b 2}\right)\left(P_{h 1}(\alpha)-P_{h 2}(\alpha)\right)}{\alpha-\alpha\left(1-P_{b 2}\right)^{2} P_{h 2}(\alpha)}
\end{aligned}
$$

where, from (16) and (19), we have

$$
2 \int_{R / 2}^{R} \delta_{h}(z) d z=\lambda_{h}-\lambda_{h c}
$$

Note that in deriving (20) and (23) we have not made any assumption on the behavior $\delta_{h}(z)$ as a function of $z$. Finally, by substituting (20) into (23), we have

$$
\begin{aligned}
\frac{\lambda_{h}}{\lambda}= & \frac{2}{3}\left(1-P_{b 1}\right)\left\{\frac{P_{h 1}(\alpha)}{1-\left(1-P_{b 2}\right) P_{h 2}(\alpha)}\right. \\
& \left.+\frac{1-P_{h 1}(\alpha)+\left(1-P_{b 2}\right)\left(P_{h 1}(\alpha)-P_{h 2}(\alpha)\right)}{\alpha-\alpha\left(1-P_{b 2}\right)^{2} P_{h 2}(\alpha)}\right\} .
\end{aligned}
$$

According to [14] and [24], the average number of handover requests per call attempt arrived at the system, $n_{h}$, can be related to $\lambda_{h} / \lambda$ as

$$
n_{h}=\frac{\lambda_{h}}{\lambda} \quad \frac{\text { handover requests }}{\text { call attempt }} .
$$

Parameter $n_{h}$ is a useful measure of the degree of mobility of the environment. If $P_{b 1}=P_{b 2}=0$, the value of $n_{h}$ given by (25) is maximum and equal to $4 /(3 \alpha)$; in particular, in the IRIDIUM mobility case $\left(R=212.5 \mathrm{~km}, V_{\text {trk }}=26600\right.$ $\mathrm{km} / \mathrm{h}$ ), on average, 4.9 handovers are required per call with $T_{m}=3 \mathrm{~min}$. It is important to point out that the proposed 
mobility model makes a difference to the results with respect to a simplified model where all MS's cross a fixed distance in a cell [14], [15]. Let us consider a cellular system equivalent to that obtained with hexagonal cells in Fig. 1, but where we eliminate the seam by using rectangular cells: these cells have the following sizes: $\sqrt{3} R \times 1.5 R$ and have the same area of hexagonal cells. Each MS crosses in a cell a distance $\sqrt{3} R$ from border to border. In this case we use the study carried out in [14] and [15]: if $P_{b 1}=P_{b 2}=0, n_{h}=1 / \alpha(=3.6$ handovers/call with IRIDIUM data and $T_{m}=3 \mathrm{~min}$ ). Hence, this simplified model underestimates the handover rate and, then, call blocking probabilities.

Among all arrived calls, we have both blocked calls (with probability $P_{b 1}$ ) and calls admitted into the network (with probability $1-P_{b 1}$ ). The average number of handover requests per call admitted in the network, $n_{h}^{\prime}$, can be related to the average number of handover per call attempt $n_{h}$ by taking into account that the quantity $1-P_{b 1}$ is the average number of accepted calls per call arrived. Therefore, if we multiply $n_{h}^{\prime}$ by $1-P_{b 1}$, we obtain $n_{h}$. Hence, the following relationship is valid:

$$
n_{h}^{\prime}=\frac{n_{h}}{1-P_{b 1}} \quad \frac{\text { handover requests }}{\text { call accepted }} .
$$

It is easy to verify that if an accepted call originates, on average, $n_{h}^{\prime}$ handover requests, and if at each request the call may be dropped with probability $P_{b 2}$, the overall call dropping probability is [14]

$$
P_{\text {drop }}=n_{h}^{\prime} P_{b 2} \text {. }
$$

Finally, probability $P_{n s}$ can be derived as [14]

$$
P_{n s}=P_{b 1}+\left(1-P_{b 1}\right) P_{\text {drop }} \text {. }
$$

\section{Channel Allocation Techniques}

In this paper, we assume that the channel allocation techniques have to fulfill the following constraint: two different cells on the earth may reuse the same channel provided that they are at a suitable distance, called reuse distance $D$ which allows tolerable levels for the cochannel interference. As stated before, we consider FCA and DCA techniques. A short description of both strategies is given below.

\section{A. Fixed Channel Allocation}

With the FCA technique, a set of channels is permanently assigned to each cell, according to the allowed reuse distance $D$. A call can only be served by an available channel (if any) belonging to the set of the cell. If an arriving call does not find any free nominal channel in its cell, the call is blocked and lost.

For uniform traffic conditions, the full set of $M$ channels is divided into equal groups each composed by $S$ channels [26]

$$
S=\frac{M}{K}, \quad \text { where } \quad K=\frac{D^{2}}{3 R^{2}} .
$$

In (30), parameter $K$ is the number of cells that form the FCA cluster [26]. The spatial repetition of this cluster assures the territory coverage as in a mosaic.
The use of FCA in a situation with nonuniform traffic requires a complex network planning in order to assign more capacity in the cells where a higher traffic is expected. In the LEO contest under consideration, such planning is meaningless because the traffic offered to a given cell is unpredictable due to the fast satellite motion with respect to the earth. This is the reason why a more suitable solution for LEO-MSS's is given by the DCA approach, as described below.

\section{B. Dynamic Channel Allocation}

A DCA strategy allows that any system channel can be temporarily assigned to any cell, provided that the constraint on the reuse distance $D$ is fulfilled. Let $x$ be the cell of the arrival, $I(x)$ the set of interfering cells with $x$ (i.e., those cells that lie at a distance less than $D$ from $x$ ), and $\Lambda(x)$ the set of available channels in $x$ [i.e., those channels that are not used either in $x$ or in the cells belonging to $I(x)$ ].

The DCA technique considered here has been introduced in [14]; it selects the channel $i^{*}$ to be allocated in the cell of the call arrival $x$ according to the following minimum cost criterion:

$$
C_{x}\left(i^{*}\right)=\min _{i \in \Lambda(x)}\left\{C_{x}(i)\right\} .
$$

The cost function $C_{x}(i)$ used in (31) has been defined as follows:

$$
C_{x}(i) \triangleq q_{x}(i)+\sum_{k \in I(x)}\left\{C_{x}(k, i)\right\} \quad \forall i \in \Lambda(x)
$$

where the cost contribution for channel $i \in \Lambda(x)$, due to the interfering cell $k \in I(x), C_{x}(k, i)$, is given by

$$
C_{x}(k, i)=v_{k}(i)+2\left(1-q_{k}(i)\right) \quad \forall k \in I(x)
$$

and

$$
\begin{aligned}
& v_{k}(i)= \begin{cases}1, & \text { if } i \in \Lambda(k) \\
0, & \text { otherwise },\end{cases} \\
& q_{k}(i)= \begin{cases}0, & \text { if } i \in F_{D}(k) \\
1, & \text { otherwise. }\end{cases}
\end{aligned}
$$

This DCA technique selects (whenever possible) channels belonging to $F_{D}(x)$, i.e., the set of channels that are assigned to $x$ by FCA. Otherwise, the DCA strategy selects in $\Lambda(x)$ the channel that becomes locked in the minimum number of interfering cells of $x$. Finally, if $\Lambda(x)=\emptyset$, the call is blocked.

In order to enhance the DCA performance, whenever a call termination occurs in a cell $x$ (due to either the physical end of a call or a handover), a channel is released in $x$ according to a deallocation criterion with a cost-function complementary to that used in the allocation phase [14]. The deallocation cost-function selects (to be freed in $x$ ) the channel $j^{*}$ that becomes available in the greatest number of interfering cells and, possibly, a channel that does not belong to the FCA pattern of $x$. If channel $j^{*}$ differs from channel $j^{\prime}$ on which the call is actually ended, the call in progress in $x$ on channel $j^{*}$ must be rearranged on channel $j^{\prime}$. Further details on this DCA technique are given in [14]. 


\section{The Queuing of HANDOVER ReQuests}

Let us consider an MS with a call in progress that leaves cell $x$ and enters an adjacent cell $y$; there is an area where this MS can receive a signal with an acceptable power level from both cells; this is the so-called overlap area. The time the MS spends to cross the overlap area $t_{w \text { max }}$ can be used to queue the related handover request if no channel is free in cell $y$. An interbeam handover strategy based on the queuing of handover requests $(\mathrm{QH})$ is essential to meet the ITU-T requirements for $P_{\text {drop }}$, as shown in Section II.

In the following, FCA NPS (DCA NPS) will denote the fixed (dynamic) channel allocation technique with no prioritization scheme (NPS) for handover requests; whereas FCA-QH (DCA-QH) will denote the fixed (dynamic) channel allocation technique with the queuing of handover requests.

\section{A. Overlap Areas Model}

In general, $t_{w} \max$ is a random variable that depends on both the degree of overlap between adjacent beams (i.e., antenna characteristics and satellites orbital configuration), the signal strength, propagation conditions (i.e., the mobile environment: rural, urban, etc.), and the MS motion direction as regards the cellular layout.

Let us assume that, due to beam-forming, spot-beam footprints are disposed on the earth according to a hexagonal regular layout (side $R$ ) and they have a circular coverage with radius $R^{\prime}$. In the literature, the possible values for the ratio $R^{\prime} / R$ range from 1 to 1.5 [27]. Obviously, the greater this ratio is, the larger the overlap area is and then the better the queuing technique performance is. In this paper, the minimum possible extension of the overlap area has been considered: $R=R^{\prime}$ (see Fig. 1).

Once the position of the MS at the call arrival instant is defined, an offset $z$ is assigned to this MS in the source cell. Due to both the regular cellular layout and the mobility assumptions, the distance $o(z)$ covered by the MS in the overlap area remains the same for any handover request, it does not matter if it originates from a source or a transit cell. For an MS crossing the overlap area at the offset $z$, parameter $t_{w \max }$ is obtained as

$$
t_{w \max }=\frac{o(z)}{V_{t r k}} .
$$

Note that, according to the assumptions made, the randomness of $t_{w \text { max }}$ only depends on the offset $z$ of the related MS that crosses the cellular network.

We assume circular spot-beam footprints on the earth with radius $R$ that give overlap areas as shown in Fig. 1. Due to the deterministic nature of the relative MS-satellite motion in LEO-MSS's, we consider that calls generated in the overlap area are automatically addressed to the destination spot-beam in order to avoid these calls generating handover requests soon after being served. Hence, the term cell denotes a region where all new calls are managed by the same spot-beam. The study of overlap areas entails a curvilinear cell shape (see the shaded region in Fig. 1), instead of the hexagonal one, previously assumed in the mobility model. Let $r(z)$ denote the distance crossed by an MS in a curvilinear cell at the offset $z$

$$
r(z)=2 \sqrt{R^{2}-z^{2}} .
$$

The maximum distance covered in a curvilinear cell before giving rise to a handover request $h(z)$ is

$$
h(z)=r(z)-o(z)
$$

where $o(z)$ is given by

$$
o(z)= \begin{cases}2 \sqrt{R^{2}-z^{2}}-\sqrt{3} R, & \text { if }|z| \leq \frac{R}{2} \\ \sqrt{R^{2}-z^{2}}-\frac{\sqrt{3}}{2} R & \\ +\sqrt{R^{2}-\left(|z|-\frac{3}{2} R\right)^{2}}, & \text { if } R \geq|z|>\frac{R}{2} .\end{cases}
$$

Note that the area of this curvilinear cell is equivalent to that of the hexagonal cell with side $R$ (i.e., $3 \sqrt{3} R^{2} / 2$ ). Then, according to the hypothesis of uniform traffic load, $\lambda$ represents the mean arrival rate of new call attempts for both curvilinear and hexagonal cells. On the basis of the new cell shape, ${ }^{3}$ we can recompute the pdf of the offset $z$ of a new call attempt in its source cell $f^{*}(z)$ by using the same approach shown in Section III to derive the pdf $f(z)$ for hexagonal cells

$$
f^{*}(z)=\frac{h(z)}{3 \frac{\sqrt{3}}{2} R^{2}} .
$$

Of course, the pdf of the offset of an active MS in a transit cell is still uniform, as for hexagonal cells. In Fig. 4 the distributions $f(z)$ and $f^{*}(z)$ are compared; we may note that $f^{*}(z)$ is not significantly different from $f(z)$. Therefore, the analytical results, which have been derived in the previous sections on the basis of a hexagonal cell shape, can be also extended, with a good approximation, to the case of curvilinear cells. ${ }^{4}$ We use $f^{*}(z)$ to derive the average value of the maximum queuing time $E\left[t_{w \max }\right]$ as

$$
\begin{aligned}
E\left[t_{w \max }\right] & =\frac{E[o(z)]}{V_{t r k}} \\
& =\frac{1}{V_{t r k}} \int_{z=-R}^{z=R} o(z) f^{*}(z) d z \\
& =\alpha T_{m} \beta
\end{aligned}
$$

where $\beta$ is given by

$$
\beta=\frac{4}{9}\left(\frac{\sqrt{3}}{3} \pi-\frac{3}{2}\right) \approx 0.1394 .
$$

Parameter $\beta$ is dimensionless and it only depends on the geometric assumptions made to model the user mobility and the overlap areas.

In particular, we have $E\left[t_{w \max }\right] \approx 7 \mathrm{~s}$ from (40) for the IRIDIUM mobility case.

\footnotetext{
${ }^{3}$ We can rewrite the points $1-4$ of the mobility model in Section III for curvilinear cells, by substituting $h(z)$ to $d(z)$.

${ }^{4}$ Please refer to [28] for the analytical computation of all the parameters of the mobility model with curvilinear cells (i.e., $P_{H i}, n_{h}, P_{\text {drop }}$, and $\lambda_{h} / \lambda$ ) and to verify the goodness of the approximation made. Note that the use of hexagonal cells allows an easier analytical formulation of the mobility model.
} 


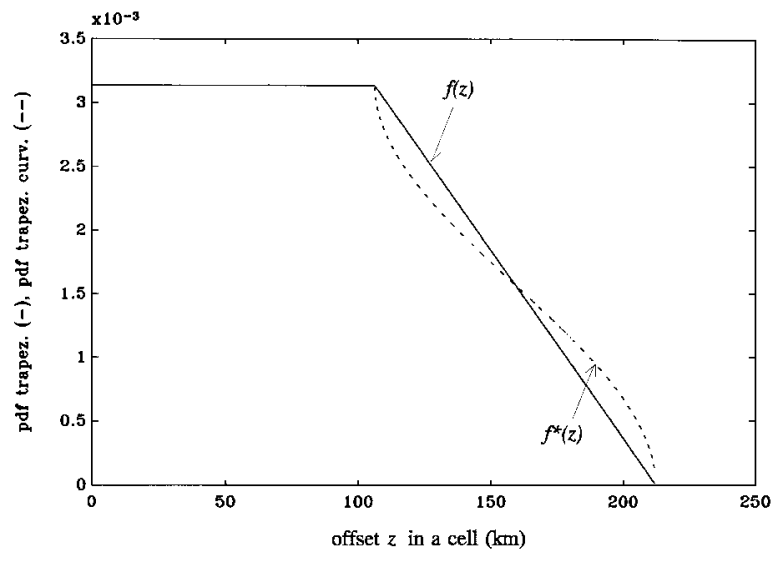

Fig. 4. Comparison between the probability density functions $f(z)$ and $f^{*}(z)$ in the IRIDIUM case.

\section{B. The Management of Handovers with the Queuing of Initially Blocked Requests}

Let us assume that a handover procedure is started as soon as an active MS goes into the overlap area between cell $x$ and cell $y$. Let $\Lambda(y)$ denote the set of free channels in the generic cell $y$. The interbeam handover request is served according to the following make-before-break arrangement.

1) If $\Lambda(y) \neq \emptyset$, the handover is immediately performed: a new channel is assigned to the MS in cell $y$ and the old channel is released in cell $x$.

2) If $\Lambda(y)=\emptyset$, the handover request is queued waiting for an available channel in cell $y$ (see Section VI-C). In the meantime, the call is served by cell $x$. A handover request leaves the queue owing to one of the three following reasons [29].

- The handover procedure is successful: the handover is performed before the call is over and its maximum queuing time has expired.

- The handover request declines: the call ends before the corresponding handover request is accomplished and its maximum queuing time has expired.

- The handover procedure fails and the associated call is dropped: the handover has not been performed within $t_{w \text { max }}$ and the call is not ended before its maximum queuing time has expired.

\section{Queuing Disciplines}

Different schemes can be applied depending on the way handover requests are ordered in the queue. The most common queuing discipline is the first input first output (FIFO) scheme [14], [15], [17], where handover requests are queued according to their arrival instants. A more complex policy is the measurement-based priority scheme (MBPS) [16], [30]. MBPS uses a nonpreemptive dynamic priority policy, where the handover priorities are defined by the power levels that the satellite receives for the related calls from their current spotbeam (we assume a network controlled handover technique, where the handover procedure is managed by the satellite [19]). The target is to serve first the calls with a more degraded link. The quality of the link is continuously monitored for each handover request in the queue to update its position.

We introduce here an alternative priority scheme called last useful instant (LUI): this discipline relies on the fact that, when a handover request is queued, the system exactly estimates its $t_{w \text { max. }}$ A new request is stored in a queue position before (after) all handover requests having a greater (lower) residual value of $t_{w \max }$ [31]. In such a way, the system tries to serve first the most urgent handover request. We can note that the ideal LUI scheme represents the best scheduling strategy for handover requests [31].

The LUI queuing scheme described above has to be regarded as an ideal scheme, because it is based on an exact estimate of time $t_{w \max }$. The performance of the MBPS technique may be made very close to that of the ideal LUI scheme if the power level measures of the queued handovers are updated according to a suitable frequency.

A practical implementation of the LUI scheme in LEOMSS's may be based on the use of a suitable positioning system which estimates the MS position at the beginning of the call and tracks the MS position during call lifetime. ${ }^{5}$ Therefore, time $t_{w \text { max }}$ can be obtained from (35), since the system knows both the relative MS-satellite motion and the cellular coverage geometry. We can consider that the MS position can be estimated by the LEO-MSS by measuring the propagation delay and Doppler frequency shift for the MS transmissions (i.e., a positioning system integrated into the LEO-MSS [32]). Using the time delay measurement, a fixed propagation delay circle is obtained on the earth. Since the Doppler frequency shift is related to the angle between the satellite velocity vector and the MS-satellite direction vector, the Doppler measurement defines a cone making a fixed angle with the satellite velocity vector. The intersection on the earth between the constant propagation delay circle and the cone identifies two points. A possible solution to solve this spatial ambiguity is to take another Doppler frequency shift measurement from a second satellite in visibility (this solution adds some constraints on the satellite constellation).

It is important to highlight that this implementation of the LUI strategy in LEO-MSS's appears to be less complex than the MBPS approach because it avoids the continuous monitoring of the signal level received by the satellite for each call with a queued handover request and, hence, the continuous ranking of the queued handover requests.

\section{Effectiveness of the LUI Queuing Scheme}

The efficiency of the LUI queuing discipline as regards the FIFO one primarily depends on the spreading degree of the distribution of $t_{w \text { max }}$ around its average value. As a matter of fact, if this distribution tends to a deterministic value, the LUI discipline approaches the FIFO one. Note that the distribution of $t_{w \text { max }}$ depends on the hypotheses made on both mobility and overlap areas.

\footnotetext{
${ }^{5}$ Once a first MS position measurement has been taken, the MS position can be tracked by estimating position variations on the basis of satellite ephemerides (this is possible because, in the LEO case, the relative satelliteMS motion is dominated by the satellite motion).
} 
Moreover, the efficiency of the LUI discipline also depends on both the mobility coefficient $\alpha$ and the congestion degree of the system. If the user mobility decreases, the time spent in the overlap area increases; then, the ordering made by the LUI scheme among the queued handover requests becomes more efficient. In addition to this, if the congestion of a cell decreases, on average, the occupancy of the handover queue decreases, so the queuing discipline has a reduced impact on system performance.

As for the management of the queued handover requests, FCA-QH and DCA-QH are significantly different, and this has an impact on the performance of the LUI scheme as regards the FIFO one, as shown in Section VIII.

\section{ANALYSIS OF FCA-QH WITH DIFFERENT QUEUING STRATEGIES}

In this section, an analytical approach for evaluating the FCA-QH performance is considered; it is based on the following assumptions and approximations.

- $S$ channels are assigned per cell according to (30).

- New call arrivals and handover attempts are two independent Poisson processes, ${ }^{6}$ with mean rates $\lambda$ and $\lambda_{h}$, with $\lambda_{h}$ related to $\lambda$ by (25).

- The statistical distribution of the channel holding time in a cell (both for new call arrivals and handovers) is approximated by an exponential distribution with mean value, $1 / \mu$, obtained as

$$
\begin{aligned}
\frac{1}{\mu}= & \frac{\lambda\left(1-P_{b 1}\right)}{\lambda\left(1-P_{b 1}\right)+\lambda_{h}\left(1-P_{b 2}\right)} E\left[t_{H 1}\right] \\
& +\frac{\lambda_{h}\left(1-P_{b 2}\right)}{\lambda\left(1-P_{b 1}\right)+\lambda_{h}\left(1-P_{b 2}\right)} E\left[t_{H 2}\right]
\end{aligned}
$$

where $E\left[t_{H 1}\right]$ and $E\left[t_{H 2}\right]$ are derived from (15).

- The maximum waiting time is approximated as a random variable exponentially distributed, with expected value $1 / \mu_{w}$ given by (40).

- The queue length is infinite.

From above, we have that each cell can be modeled as an $M / M / S$ queuing system with nonhomogeneous arrival rates [14], [16], [17] ( $M$ : Poisson arrival process $M$ : service time exponentially distributed/ $S$ : number of channels assigned per cell), as shown in Fig. 5. The state of this queuing system is given by the sum of the number of calls in service and the number of queued handover requests. Whenever the system is in a state $n$ less than $S$, the gross arrival rate is $\lambda+\lambda_{h}$; while, if the state is greater than or equal to $S$ (i.e., all channels are busy), the gross arrival rate is $\lambda_{h}$. Moreover, we have considered that a call may end in the overlap area before obtaining service and before its maximum queuing time has expired [29]. Accordingly, the Markov chain in Fig. 5 contains additional death rates $i \mu$ for the states $S+i$, with $i=1,2, \cdots$,

\footnotetext{
${ }^{6} \mathrm{~A}$ generalization of this analytical study to different arrival processes for handover requests, such as a bursty arrival process (e.g., suitable for modeling handover arrivals for public transportation systems), seems to be possible at the expense of a greater complexity [24]. However, in this case the main problem is to give an effective statistical characterization of the handover traffic in relation to specific scenarios. This study requires a deep investigation which is beyond the scope of this paper and has been left to a further study.
}

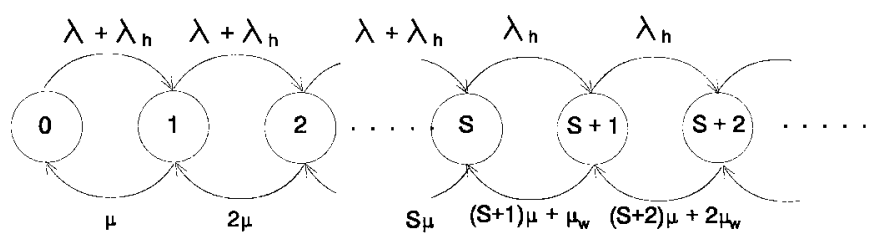

Fig. 5. The queuing system for FCA-QH.

as regards the model appeared in [14]. The Markov chain shown in Fig. 5 is valid for both FIFO and LUI disciplines. The probability of state $n, P_{n}$, is

$$
\begin{aligned}
& P_{n}=
\end{aligned}
$$

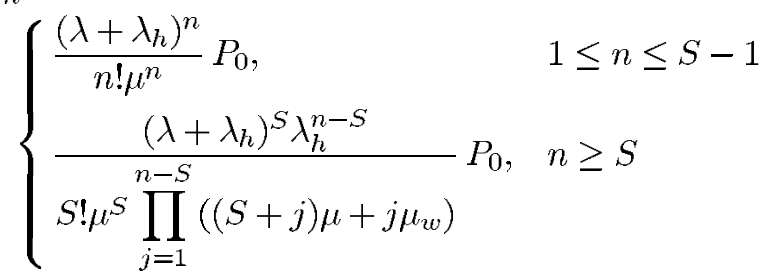

where the idle system probability $P_{0}$ is

$$
\begin{aligned}
P_{0}=\left\{\sum_{n=0}^{S-1}\left[\frac{\left(\lambda+\lambda_{h}\right)^{n}}{n ! \mu^{n}}\right]\right. \\
\left.+\sum_{n=S}^{\infty}\left[\frac{\left(\lambda+\lambda_{h}\right)^{S} \lambda_{h}^{n-S}}{S ! \mu^{S} \prod_{j=1}^{n-S}\left((S+j) \mu+j \mu_{w}\right)}\right]\right\}^{-1} .
\end{aligned}
$$

New call arrivals are blocked when all the available channels are in use, i.e., when the queuing system is in the state $n \geq S$. Therefore, $P_{b 1}$ results in

$$
P_{b 1}=\sum_{N=S}^{\infty} P_{n} .
$$

The blocking parameter $P_{b 1}$ does not depend on the queuing discipline. This statement has been verified by means of computer simulations (see the next section). Whereas $P_{b 2}$ depends on the queuing policy.

With the FIFO queuing discipline, $P_{b 2}$ can be derived by the approach outlined in [14] and [17] and by taking the following into account.

1) $P_{b 2}$ must contain, as a multiplying factor, the probability that the call, with a queued handover request, does not end before $t_{w \text { max }}$ has expired, $P_{u h}$. According to the exponential distributions for the maximum queuing time and the channel holding time, $P_{u h}$ is

$$
P_{u h}=\frac{\mu_{w}}{\mu+\mu_{w}} .
$$

2) State probabilities are given by (43) and (44). 
3) We consider the additional departure rates $i \mu$ for states $S+i$ with $i=1,2, \cdots$, due to calls that end in the overlap area before accomplishing the related handover procedures.

Hence, we have

$$
\begin{aligned}
& P_{b 2}=\frac{\mu_{w}}{\mu+\mu_{w}} \sum_{n=S}^{\infty} P_{n}\left\{1-\frac{S \mu}{S \mu+\mu_{w}}\right. \\
&\left.\cdot \prod_{j=1}^{n-S}\left[1-\frac{\mu_{w}}{S \mu+\mu_{w}}\left(\frac{\mu_{w}}{\mu+\mu_{w}} \frac{1}{2}\right)^{j}\right]\right\} .
\end{aligned}
$$

With the LUI strategy, each handover request in the queue reaches the head of the queue, unless the request leaves the queue because the related call is ended. Hence, only the handover request at the head of the queue may fail. Therefore, the failure probability for a handover request that initially reaches the queue at position $n(>S)$ does not depend on $n$; let us denote by $P_{b 2 \mid S}$ this handover failure probability. Then, by using (45), the following result is obtained:

$$
P_{b 2}=\sum_{n=S}^{\infty} P_{n} P_{b 2 \mid S}=P_{b 2 \mid S} P_{b 1}
$$

$P_{b 2 \mid S}$ takes into account two joint and independent events.

1) The call, whose handover request is at the head of the queue, does not end before its maximum queuing time has expired; the probability of this event is $P_{u h}$.

2) None of the $S$ channels of the cell becomes free before the maximum queuing time has expired. Let us denote the probability of this event by $P_{f}$. According to the exponential distributions for the maximum queuing time and the channel holding time, we have

$$
P_{f}=\frac{\mu_{w}}{S \mu+\mu_{w}} .
$$

In conclusion, in the LUI case, $P_{b 2}$ is given by

$$
P_{b 2}=P_{b 1} \frac{\mu_{w}}{\mu+\mu_{w}} \frac{\mu_{w}}{S \mu+\mu_{w}} .
$$

Note that a recursive approach is necessary to compute $P_{b 1}$ and $P_{b 2}$ (for both queuing disciplines) as functions of $\lambda$, because $\lambda_{h}$ depends on both $P_{b 1}$ and $P_{b 2}$, according to (25). In order to speed up the convergence, the iterative method is not based on the two parameters $P_{b 1}$ and $P_{b 2}$, as proposed in [14], but only on parameter $n_{h}=\lambda_{h} / \lambda$, which is a function of $P_{b 1}$ and $P_{b 2}$.

We start the iterations with the value of $n_{h}$ obtained from (25) and (26) with $P_{b 1}=P_{b 2}=0$ (this is the maximum value of $n_{h} ; n_{h}$ decreases for increasing values of $P_{b 1}$ or $\left.P_{b 2}\right)$. With such a value of $n_{h}, \mu$, and $P_{n}$ for $n=0,1, \cdots$, are computed according to (42)-(44). These values are used to derive $P_{b 1}$ and $P_{b 2}$ and, then, the new value of $n_{h}$. This value is averaged with that used at the previous step. A new iteration starts with this mean value of $n_{h}$. The iterative method is stopped when the relative difference between the $n_{h}$ values computed in two subsequent steps is below a given threshold (i.e., $10^{-3}$ ). Finally, we can derive $P_{n s}$ from (29).

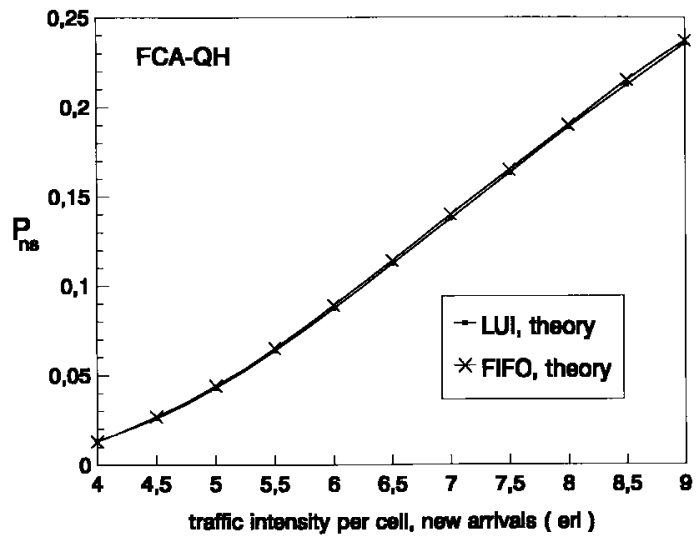

Fig. 6. Theoretic comparison between the performance of FIFO and LUI queuing disciplines for FCA-QH in terms of $P_{n s}$ (IRIDIUM case, $S=10$ ).

Fig. 6 highlights the analytical results concerning FCAQH with FIFO and LUI schemes in the IRIDIUM case with $S=10$. We note that the LUI strategy slightly outperforms the FIFO one. This result can be justified by considering that the time spent in the overlap area by an MS is so small (on average $7 \mathrm{~s}$ ) that, in the queue of each cell, there is not a significant difference between serving either the most urgent handover request or the oldest one. A different impact of the LUI scheme will be highlighted in the next section by considering DCA-QH.

\section{Simulation Results}

In obtaining our simulation results, we have removed all the simplifications made in the previous section in order to analytically derive the FCA-QH performance. In particular, we have used the models for mobility and overlap areas shown in Sections III and VI, respectively. Circular cells are considered for the resource management strategies based on handover queuing; whereas hexagonal cells are used for assignment strategies with the NPS scheme. The parameter values used in the simulations are:

- the average call duration is $T_{m}=3 \mathrm{~min}$;

- the reuse distance is $D=\sqrt{21} R$;

- the simulated cellular network is parallelogram shaped and folded onto itself ${ }^{7}$ with seven cells per side,

- a number of 70 channels is available to the system; then, ten channels/cell are available with FCA;

- the IRIDIUM mobility case is considered (i.e., $\alpha \approx 0.27$ ).

Moreover, we have assumed an infinite queue capacity for handover requests. Since theoretic results in Fig. 6 have revealed that the performance difference between LUI and FIFO queuing schemes is very little, simulation results have been gathered after very long simulation runs that allow 5\% confidence intervals for the blocking results [33].

In Fig. 7(a) and (b), we can note that the analytical predictions for FCA-QH with both queuing disciplines give a conservative estimate of $P_{n s}$ in comparison with simulation results. This difference is exclusively due to the simplifications

\footnotetext{
${ }^{7}$ The parallelogram-shaped network we have simulated has been folded onto itself [15]. Therefore, handover requests are naturally generated for border cells and each cell has a complete set of adjacent cells as in the real three-dimensional global coverage satellite cellular system.
} 


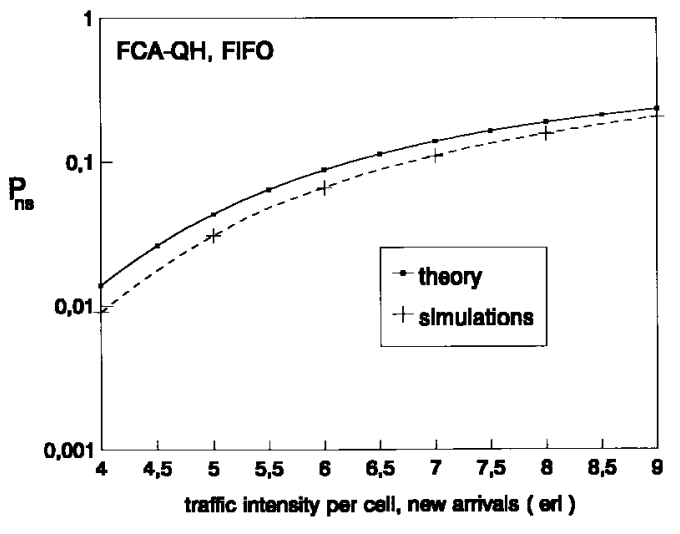

(a)

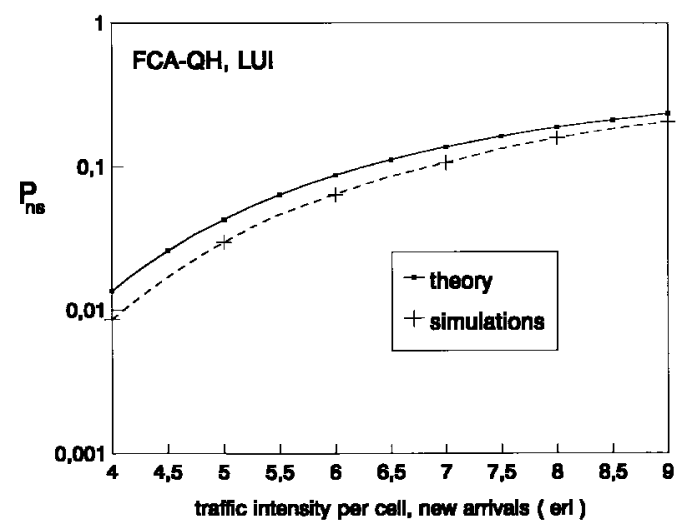

(b)

Fig. 7. (a) FCA-QH with FIFO queuing discipline: comparison between simulation and analytical predictions (IRIDIUM case, $S=10$ ). (b) FCA-QH with LUI queuing discipline: comparison between simulation and analytical predictions (IRIDIUM case, $S=10$ ).

assumed in our analysis for both the pdf of $t_{w \max }$, the handover arrival process, and the pdf of the channel holding time.

Let us remember that the ITU-T requirements for the quality of service parameters are $P_{b 1} \leq 10^{-2}$ and $P_{\text {drop }} \leq 5 \cdot 10^{-4}$ [22]. Referring to the IRIDIUM mobility conditions, we have $n_{h}^{\prime}<5$ handovers/call [see (27)]. Hence, on the basis of (28), the ITU-T requirement on $P_{\text {drop }}$ is fulfilled if $P_{b 2} \leq 10^{-4}$. We are interested in evaluating the maximum traffic intensity per cell due to new call arrivals (i.e., $\lambda T_{m}$ ) that allows $P_{b 1} \leq 10^{-2}$ and $P_{b 2} \leq 10^{-4}$. This traffic load will be converted into capacity per cell (i.e., the maximum number of users per cell) by assuming that each user generates 0.025 erl of traffic [14]. Let us comment on the simulation results shown in Figs. 8-10.

\section{A. DCA versus FCA}

From Figs. 8 and 9 we note that the more critical requirement (i.e., the requirement that mostly limits the system capacity) is that on $P_{b 2}$. None of the considered FCA techniques (i.e., FCA NPS, FCA-QH FIFO, and FCA-QH LUI) fulfills the requirements on $P_{b 1}$ and $P_{b 2}$ in the traffic range under examination. According to Table I, DCA techniques attain significantly better results in terms of maximum traffic intensity per cell and capacity per cell; moreover, DCA-QH

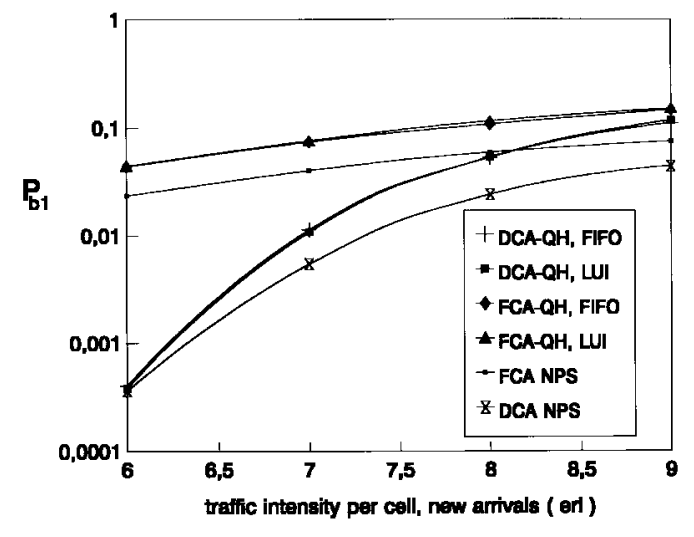

Fig. 8. $P_{b 1}$ performance for FCA NPS and DCA NPS, FCA-QH and DCA-QH both FIFO and LUI (IRIDIUM case).

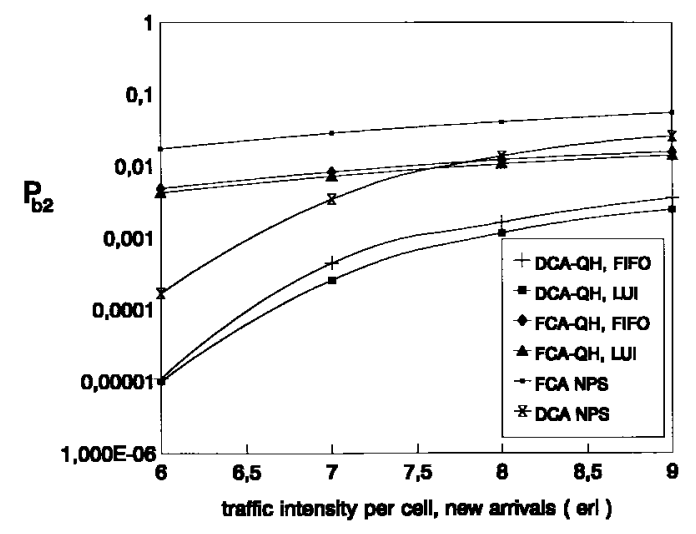

Fig. 9. $P_{b 2}$ performance for FCA NPS and DCA NPS, FCA-QH and DCA-QH both FIFO and LUI (IRIDIUM case).

LUI achieves the highest system capacity among the resource management strategies considered in this paper.

\section{B. NPS versus $Q H$}

As regards the NPS scheme, the QH strategy (regardless of both the queuing discipline and the channel allocation technique used) allows a significant reduction of $P_{b 2}$ at the expense of an increased value of $P_{b 1}$. The advantages of $\mathrm{QH}$ schemes are particularly evident in terms of $P_{n s}$, as shown in Fig. 10.

\section{FIFO versus LUI}

Numerical results derived by means of computer simulations for both FCA-QH and DCA-QH have confirmed that $P_{b 1}$ (Fig. 8) is independent of the adopted queuing discipline (i.e., FIFO or LUI).

Let us refer to the behavior of $P_{b 2}$ shown in Fig. 9. With FCA-QH, the advantages of the LUI discipline with respect to the FIFO one are practically negligible. Whereas in the case of DCA-QH, the LUI scheme permits the reduction of $P_{b 2}$ as regards the FIFO policy. The reason for these different behaviors has to be searched in the way the handover queuing is managed by FCA-QH and DCA-QH. With FCA-QH, each cell has its queue, whereas DCA-QH requires that the system 


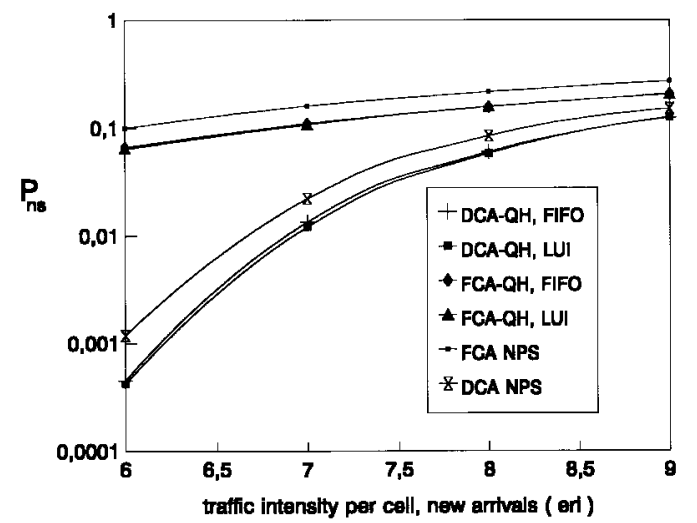

Fig. 10. $P_{n s}$ performance for FCA NPS and DCA NPS, FCA-QH and DCA-QH both FIFO and LUI (IRIDIUM case).

TABLE I

DCA Techniques Performance in Terms of MAXIMUM TRAFFIC INTENSITY AND CAPACITY

\begin{tabular}{c|c|c}
\hline \hline DCA technique & $\begin{array}{c}\text { maximum traffic intensity per cell due to } \\
\text { new call anivals (er) }\end{array}$ & $\begin{array}{c}\text { capacity per cell } \\
\text { (\#users/ceil) }\end{array}$ \\
\hline DCA NPS & 6 & 240 \\
\hline DCA-QH FIFO & 6.6 & 264 \\
\hline DCA-QH LUI & 6.8 & 272 \\
\hline \hline
\end{tabular}

manages a virtual global queue ${ }^{8}$ formed by the handover requests waiting for service in all the cells. This global queue contains a greater number of handover requests than each single queue of FCA-QH. Hence, a specific ordering discipline has a greater impact on DCA-QH rather than on FCA-QH. This is a further advantage of the DCA approach as regards the FCA one.

\section{Nonuniform Traffic Loads}

In order to highlight that the advantages of the DCA scheme over the FCA approach can be enhanced in the case of nonuniform traffic, we have considered here the traffic model for LEO-MSS's proposed in [25]. In particular, the study presented in [25] has allowed us to forecast a traffic distribution on the earth that has been divided in areas of $5^{\circ}$ longitude $\times 5^{\circ}$ latitude. This traffic distribution [see Fig. 11(a)] has been obtained by assuming $T_{m}=2 \mathrm{~min}$. Based on this result, Fig. 11(b) shows a performance comparison in terms of $P_{n s}$ between DCA-QH and FCA-QH with the FIFO queuing for handover requests. These results have been obtained by assuming the system parameter values given at the beginning of this section (with the exception of $T_{m}=2 \mathrm{~min}$ ). From Fig. 11(b) it is evident that the advantages of DCA$\mathrm{QH}$ with respect to FCA-QH can be magnified in the case of nonuniform traffic.

\footnotetext{
${ }^{8}$ Since with DCA NPS (and DCA-QH) the availability of a channel in a cell depends on the state of this channel in all the interfering cells, all the cells are interdependent in managing the queued handover requests.
}

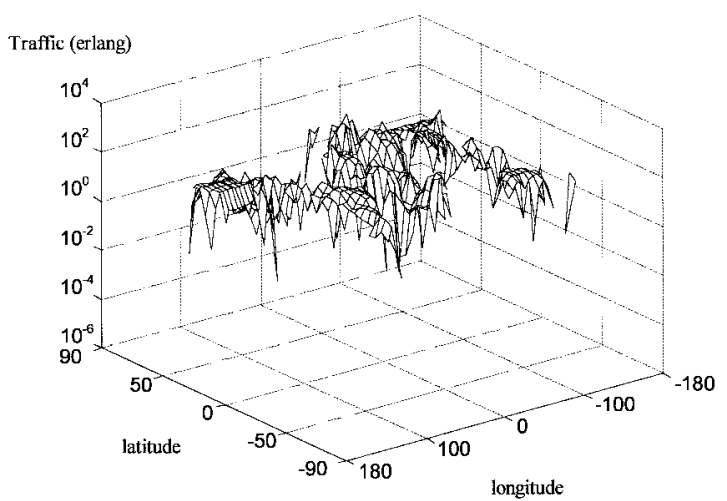

(a)

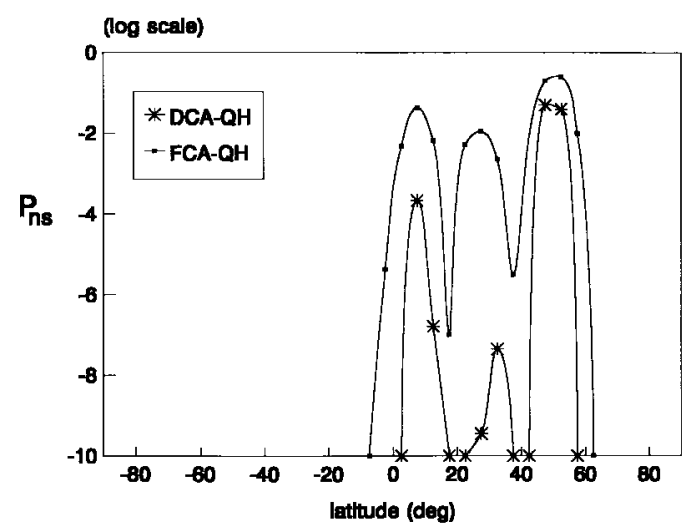

(b)

Fig. 11. (a) Traffic forecast on the earth for future MSS's; each point is the traffic in erlang related to a $5^{\circ}$ longitude $\times 5^{\circ}$ latitude wide area. (b) Comparison of DCA-QH and FCA-QH in terms of $P_{n s}$ for the traffic situation around the meridian of $10^{\circ} \mathrm{E}$ longitude.

\section{CONCLUSIONS}

This paper has presented a mobility model suitable for performance evaluation of channel allocation strategies in LEO-MSS's. Such a mobility model permits the removal of the approximation made in previous works of neglecting the motion of MS's along the seam of the cellular network and, hence, the increase of the accuracy of the performance evaluation. We have discussed this aspect which is of particular importance in LEO-MSS's, where interbeam handovers are very frequent during call lifetime.

In order to reduce the handover failure probability, the service of handover requests must be prioritized as regards that of new call attempts. Therefore, we have considered two different handover queuing schemes: i.e., FIFO and LUI. In particular, for the novel LUI approach a practical implementation has also been discussed and compared with that of the MBPS queuing scheme, previously presented in the literature.

Performance evaluations and comparisons have been carried out in terms of QoS parameters specified in the ITU-T Recommendation E.771. In particular, we have proved by simulations that the DCA technique outperforms the corresponding FCA one, it does not matter which handover prioritization scheme is adopted. Moreover, we have briefly proved that this advantage is increased with nonuniform traffic. Finally, we have shown that DCA-QH with the LUI queuing discipline allows a high 
system capacity and, hence, it is a very attractive scheme for LEO-MSS's.

\section{ACKNOWLEDGMENT}

The authors would like to acknowledge the anonymous reviewers and Prof. D. Everitt for providing useful comments that have enhanced the technical quality and clarity of this paper.

\section{REFERENCES}

[1] "Phones into orbit," The Economist, Mar. 27-28, 1992.

[2] E. Del Re, "A coordinated European effort for the definition of a satellite integrated environment for future mobile communications," IEEE Commun. Mag., vol. 34, pp. 98-104, Feb. 1996.

[3] M. Mohorcic, G. Kandus, E. Del Re, and G. Giambene, "Performance study of an integrated satellite/terrestrial mobile communication system," Int. J. Satellite Commun., vol. 14, no. 5, pp. 413-425, Sept./Oct. 1996.

[4] L.-R. Hu and S. S. Rappaport, "Personal communication systems using multiple hierarchical cellular overlays," IEEE J. Select. Areas Commun., vol. 13, pp. 406-415, Feb. 1995.

[5] P. W. Baier, P. Joung, and A. Klein, "Taking the challenge of multiple access for third-generation cellular mobile radio systems-A European view," IEEE Commun. Mag., vol. 34, pp. 82-89, Feb. 1996.

[6] G. Maral, J.-J. De Ridder, B. G. Evans, and M. Richharia, "Low Earth orbit satellite systems for communications," Int. J. Satellite Commun., vol. 9, pp. 209-225, 1991.

[7] A. Ganz, Y. Gong, and B. Li, "Performance study of low Earthorbit satellite systems," IEEE Trans. Commun., vol. 42, pp. 1866-1871, Feb.-Apr. 1994.

[8] J. L. Grubb, "IRIDIUM overview," IEEE Commun. Mag., vol. 29, no. 11, Nov. 1991.

[9] Official Web site of the TELEDESIC system with address: http://www.teledesic.com.

[10] F. Ananasso and F. D. Priscoli, "The role of satellites in personal communication services," IEEE J. Select. Areas Commun., vol. 13, pp. 180-196, Feb. 1995.

[11] L. J. Cimini, G. J. Foschini, C.-L. I, and Z. Miljanic, "Call blocking performance for dynamic channel allocation in microcells," IEEE Trans. Commun., vol. 42, pp. 2600-2607, Aug. 1994.

[12] S. Tekinay and B. Jabbari, "Handover and channel assignments in mobile cellular networks, "IEEE Commun. Mag., vol. 29, pp. 42-46, Nov. 1991.

[13] E. Del Re, R. Fantacci, and G. Giambene, "Performance analysis of a dynamic channel allocation technique for satellite mobile cellular networks," Int. J. Satellite Commun., vol. 12, pp. 25-32, Jan./Feb. 1994.

[14] _ _Efficient dynamic channel allocation techniques with handover queuing for mobile satellite networks," IEEE J. Select. Areas Commun., vol. 13, pp. 397-405, Feb. 1995.

[15] _ , "An efficient technique for dynamically allocating channels in satellite cellular networks," in Proc. IEEE GLOBECOM'95, Singapore, Nov. 13-17, 1995, pp. 1624-1628.

[16] S. Tekinay and B. Jabbari, "A measurement-based prioritization scheme for handovers in mobile cellular networks," IEEE J. Select. Areas Commun., vol. 10, pp. 1343-1350, Oct. 1992.

[17] D. Hong and S. S. Rappaport, "Traffic model and performance analysis for cellular mobile radio telephone systems with prioritized and nonprioritized handoff procedures," IEEE Trans. Veh. Technol., vol. VT-35, pp. 77-92, Aug. 1986.

[18] S. S. Rappaport, "Blocking, hand-off and traffic performance for cellular communication systems with mixed platforms," Proc. Inst. Elect. Eng., vol. 140, no. 5, pt. I, pp. 389-401, Oct. 1993.

[19] Y.-B. Lin, S. Mohan, and A. Noerpel, "Queuing priority channel assignment strategies for PCS hand-off and initial access," IEEE Trans. Veh. Technol., vol. 43, pp. 704-712, Aug. 1994.

[20] _ , "PCS channel assignment strategies for hand-off and initial access," IEEE Personal Commun., pp. 47-56, Third Quarter 1994.

[21] - "Queueing channel assignment strategies for PCS hand-off and initial access," in Proc. ICUPC'94, San Diego, CA, Sept. 27-Oct. 1, 1994, pp. 365-369.
[22] ITU-E.771 "Network grade of service parameters and target values for circuit-switched land mobile services," Blue Book, 1995.

[23] Y.-B. Lin, L.-F. Chang, and A. Noerpel, "Modeling hierarchical microcell/macrocell PCS architecture," in IEEE ICC'95, Seattle, June 1995, pp. 405-409.

[24] S. M. Ross, Stochastic Processes. New York: Wiley, 1983.

[25] R. E. Sheriff, H. F. Hu, E. Del Re, R. Fantacci, and G. Giambene, "Satellite-UMTS traffic dimensioning and resource management technique analysis," IEEE Trans. Veh. Technol., vol. 4, pp. 131-134, Nov. 1998.

[26] V. H. MacDonald, "The cellular concept," Bell Syst. Tech. J., vol. 58, pp. 15-41, Jan. 1979.

[27] T.-P. Chu and S. Rappaport, "Overlapping coverage and channel rearrangement in microcellular communication systems," in Proc. IEEE GLOBECOM'94, San Francisco, CA, Nov. 28-Dec. 2, 1994, pp. $1674-1678$.

[28] E. Del Re, R. Fantacci, and G. Giambene, "Different queuing policies for handover requests in low earth orbit mobile satellite systems," IEEE Trans. Veh. Technol., to be published.

[29] C. Purzynski and S. S. Rappaport, "Multiple call hand-off problem with queued hand-offs and mixed platform types," Proc. Inst. Elect. Eng., vol. 142, no. 1, pt. I, pp. 31-39, Feb. 1995.

[30] S. Tekinay and B. Jabbari, "Analysis of measurement based prioritization schemes for handovers in cellular networks," in Proc. IEEE GLOBECOM'92, Orlando, FL, 1992.

[31] M. Gurmundson, "Analysis of handover algorithms," in Proc. IEEE VTC'91, pp. 539-543.

[32] W. Zhao, R. Tafazolli, and B. G. Evans, "A UT positioning approach for dynamic satellite constellations," in Proc. 4th IMSC'95, Ottawa, Canada, June 6-8, 1995, pp. 251-258.

[33] D. D. Dimitrijevic and J. Vucetic, "Design and performance analysis of the algorithms for channel allocation in cellular networks," IEEE Trans. Veh. Technol., vol. 42, pp. 526-534, Nov. 1993.

[34] D. Everitt and D. Manfield, "Performance analysis of cellular mobile communication systems with dynamic channel assignment," IEEE $J$. Select. Areas Commun., vol. 7, pp. 1172-1180, Oct. 1989.

Enrico Del Re (M'78-SM'84) was born in Florence, Italy, in 1947. He received the Dr.Ing. degree in electronics engineering from the University of Pisa, Pisa, Italy, in 1971.

Until 1975, he was engaged in public administration and private firms, involved in the analysis and design of the telecommunication and air traffic control equipment and space systems. Since 1975, he has been with the Department of Electronics Engineering of the University of Florence, Florence, Italy, first as a Research Assistant, then as an Associate Professor, and since 1986, as Professor. During the academic year 1987-1988, he was on leave from the University of Florence for a nine-month period of research at the European Space Research and Technology Centre of the European Space Agency, The Netherlands. His main research interests are digital signal processing, digital transmission techniques, and communication networks, on which he has published more than 150 papers in international journals and conferences. He is the coeditor of the book Satellite Integrated Communications Networks (North-Holland, 1988) and one of the authors of the book Data Compression and Error Control Techniques with Applications (Academic, 1985). He has been the Chairman of the European Project COST 227 "Integrated Space/Terrestrial Mobile Networks" and he is now Chairman of the COST 252 project: "Evolution of Satellite Personal Communications from 2nd to Future Generation Systems." He is the head of the Digital Signal Processing and Telematics Laboratory (LENST) of the Department of Electronic Engineering of the University of Florence.

Prof. Del Re is a member of the AEI the European Association for Signal Processing (EURASIP). He has been included in the edition of Who's Who in the World. He teaches a course in digital signal processing. He received the 1988/1989 premium from the IEE (UK) for the paper "Multicarrier Demodulator for Digital Satellite Communication Systems." 
Romano Fantacci (S'82-M'87-SM'91) was born in Pistoia, Italy. He received the Dr.Ing. degree in electronics engineering from the University of Florence, Florence, Italy, in 1982 and the Ph.D. degree in telecommunication engineering in 1987.

In 1982, he joined the Electronics Engineering Department of the University of Florence, first as a Researcher in computer communications and currently as an Associate Professor of telecommunication networks. During the first stage of his research activity he worked on satellite communication systems. In particular, he has been involved in several European Space Agency (ESA) and INTELSAT advanced research projects. His present research interests involve digital communications, computer communications, queuing theory, fast packet switching, and mobile communication networks. He is the author of several publications, some of which have been published in the most acknowledged journals in communication science. He has been involved in several European (RACE) and nationwide research projects sponsored by Italian National Council of Research (CNR) and Italian Minister of Education and Research (MURST).

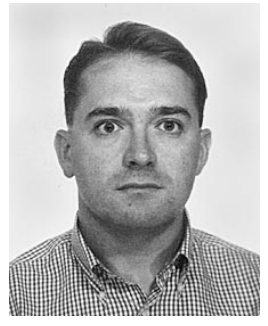

Giovanni Giambene (S'94-M'97) received the Dr.Ing. degree in electronics from the University of Florence, Florence, Italy, in 1993 and the Ph.D. degree in telecommunications and informatics in 1997.

Since 1994, he has been with the Electronic Engineering Department, University of Florence, as a Research Assistant. He was the Technical External Secretary of the European Community Project COST 227 "Integrated Space/Terrestrial Mobile Networks." He also contributed to the "Research Management" activity of the working group 3000 within the RACE Project called "Satellite Integration in the Future Mobile Network" (SAINT, RACE 2117). His research interests include mobile cellular communication networks, personal communication services, channel allocation techniques, and queuing theory. 